EPJ Web of Conferences 40, 14001 (2013)

DOI: $10.1051 /$ epjconf/20134014001

(C) Owned by the authors, published by EDP Sciences, 2013

\title{
The Effect of Pressure on Magnetic Properties of $\mathrm{KMnCr}(\mathrm{CN})_{6}$.
}

\author{
M.Vavra ${ }^{1,2}$, P.Hrabčák ${ }^{1}$, M.Zentková ${ }^{1}$, M.Mihalik $^{1}$, M.Mihalik,Jr $^{1}$, K. Csach $^{1}$ \\ ${ }^{1}$ Institute of Experimental Physics SAS, Watsonova 47, 04001 Košice, Slovakia \\ ${ }^{2}$ P.J.Šafárik University, Moyzesova 11, 04154 Košice, Slovakia
}

\begin{abstract}
In the contribution we present the effect of pressure on magnetic properties of molecule based magnet $\mathrm{KMnCr}(\mathrm{CN})_{6}$. Applied pressure affects magnetization curves only marginally. The saturation is reached at higher magnetic fields under pressure, but the effect of the pressure on the values of saturated magnetization $\mu_{\mathrm{s}}$, remnant magnetization $\mu_{\mathrm{r}}$ and coercive field $H_{\mathrm{C}}$ are almost negligible. Observed pronounced increase of the Curie temperature $T_{\mathrm{C}}$ with increasing pressure can be attributed to strengthening of antiferromagnetic superexchange interaction. Additionally we observed double magnetic transition induced by hydrostatic pressure. All pressure changes were fully reversible.
\end{abstract}

\section{Introduction}

Prussian blue analogues (PBA) form a large family of cubic systems with face-centred (fcc) crystal structure. One of the possible stoichiometries has chemical formula $\mathrm{MA}^{\mathrm{II}}\left[\mathrm{B}^{\mathrm{III}}(\mathrm{CN})_{6}\right]$ where $\mathrm{M}$ is an alkali metal cation e.g. $\mathrm{K}$ which is placed into an interstitial sites; A is $3 \mathrm{~d}$ metal which occupies all the summits and all the centres of the faces and the $\left[\mathrm{B}(\mathrm{CN})_{6}\right]$ are located at the octahedral sites. One-third of $\left[\mathrm{B}(\mathrm{CN})_{6}\right]$ vacancies are filled by $\mathrm{M}$ - an alkali metal cation; the $\mathrm{B}$ coordination sphere is left unchanged but the mean coordination sphere of $\mathrm{A}$ becomes $\mathrm{A}(\mathrm{NC})_{4}\left(\mathrm{H}_{2} \mathrm{O}\right)_{2}$ [1]. A metal ion is placed next to nitrogen atom and thus due to weak ligand field is almost always high spin. On the other hand B magnetic ion placed next to carbon atom is in strong ligand field and therefore B is always low spin. The magnetic coupling in these systems is determined by super-exchange interaction between metal ions $\mathrm{A}^{2+}$ and $\mathrm{B}^{\mathrm{III}}$ mediated through a three-dimensional network of $\mathrm{C}^{*} \mathrm{CN}$ bridges, resulting in $3 \mathrm{D}$ magnetic ordering with transition temperatures $T_{\mathrm{C}}$ up to $376 \mathrm{~K}$ [2] depending on the nature of the metal ions. The magnetic properties of PBA can be analysed within two simplifications: (i) only the superexchange interactions between the nearest neighbour metal $\mathrm{A}^{2+}$ and $\mathrm{B}^{\mathrm{III}}$ ions have to be considered; (ii) if the magnetic orbital symmetries of the metal ions are the same, the super-exchange interaction is antiferromagnetic $\left(\mathrm{J}_{\mathrm{AF}}\right)$; conversely, when their magnetic orbital symmetries are different, the super-exchange interaction is ferromagnetic $\left(\mathrm{J}_{\mathrm{F}}\right)$. This simple model has already been tested on the $\mathrm{TM}^{2+}{ }_{3}\left[\mathrm{Cr}^{\mathrm{III}}(\mathrm{CN})_{6}\right]_{2} \cdot 15 \mathrm{H}_{2} \mathrm{O}$ system, $\mathrm{TM}^{2+}$ is a $3 \mathrm{~d}$ ion [3]. The $\mathrm{Cr}^{\mathrm{III}}$ in the anion $\left[\mathrm{Cr}^{\mathrm{III}}(\mathrm{CN})_{6}\right]_{3}$. has only $\left(\mathrm{t}_{2} \mathrm{~g}\right)^{3}$ orbitals and there are six ferromagnetic (F) and nine antiferromagnetic (AF) pathways with $\left(\mathrm{t}_{2 \mathrm{~g}}\right)^{3}\left(\mathrm{e}_{\mathrm{g}}\right)^{2}$ orbitals of $\mathrm{Mn}^{2+}$ leading to overall $\mathrm{J}_{\mathrm{AF}}$ interaction. The large number of possible combinations of ions and oxidation states give rise to many competing orbital interactions, which will cause the structure to reach an overall order, either fero or ferimagnetic. Magnetic properties of PBAs are very sensitive to the effect of external parameters such as humidity, light and pressure $[1,2]$. For PBAs the effect of pressure triggered the following mechanisms: high spin low spin transition [4] or magnetic pole inversion [5] can be induced by pressure. The first work dealing with the study of pressure effect on magnetic properties was referred by Awaga et. all in [6]. The increase in Curie temperature under pressure was ascribed to the enhancement of antiferromagnetic interaction as the consequence of the exchange pathway shortening. In our earlier work we have shown that for PBA with domination of antiferromagnetic interaction the Curie temperature increases under pressure while for those with dominating ferromagnetic it remains unchanged or slowly decreases [7]. The applied pressure can slightly affect bonding angles between magnetic ions mediated by the cyano-bridge. A small deviation from the ideal value $180^{\circ}$ of the bonding angle can even reduce the strength of magnetic coupling, and results in reduction of $T_{\mathrm{C}}$ [7]. Unusually high value of magnetic Grüneisen parameter $\varepsilon$ (in the range 9.03-9.97) was reported for PBA [8]. In a phenomenological model the material-dependent parameter $\varepsilon$ relates to the intersite distances, i.e. correlates with interaction strength J. This simple fact 


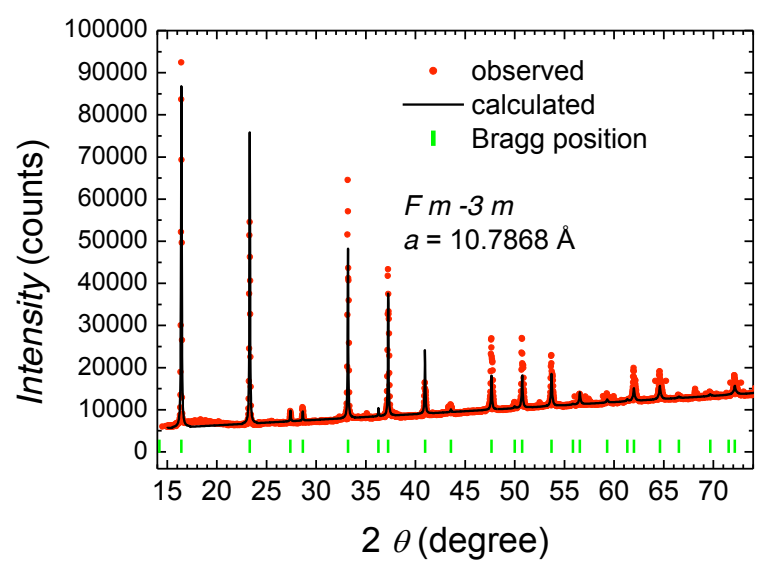

Fig.1. X-ray powder diffraction pattern of $\mathrm{KMn}[\mathrm{Cr}(\mathrm{CN}) 6]$. Experimentally measured intensities are indicated by circles and the calculated ones by the line which overlays them. The positions of all possible Bragg reflections are marked by the vertical marks.

points out to very strong magneto-structural correlation in PBA [8].

\section{Experimental}

$\mathrm{KMn}\left[\mathrm{Cr}(\mathrm{CN})_{6}\right]$ compound has been prepared by slow addition of the concentrated $\mathrm{K}_{3}\left[\mathrm{Cr}(\mathrm{CN})_{6}\right](0.3968 \mathrm{~g}$ dissolved in $10 \mathrm{ml}$ of water, $1.219 \mathrm{mmol}$ ) solution to the aqueous solution of $\mathrm{MnCl}_{2}\left(0.2413 \mathrm{~g} \mathrm{MnCl}_{2} .4 \mathrm{H}_{2} \mathrm{O}\right.$ in 10 $\mathrm{ml}$ of water, $1.219 \mathrm{mmol})$. In a few minutes, yellow precipitate has been filtrated off and washed with water. Magnetization was studied in the temperature range $8 \mathrm{~K} \leq$ $T \leq 100 \mathrm{~K}$ and magnetic fields up to $\mu_{0} H=5 \mathrm{~T}$ using a SQUID magnetometer (MPMS). Pressure was generated by a hydrostatic $\mathrm{CuBe}$ pressure cell filled with a mixture of mineral oils serving as the pressure transmitting medium and operating up to $1.2 \mathrm{GPa}$. Actual pressure was determined from the pressure dependence $T_{\mathrm{C}}(p)$ of

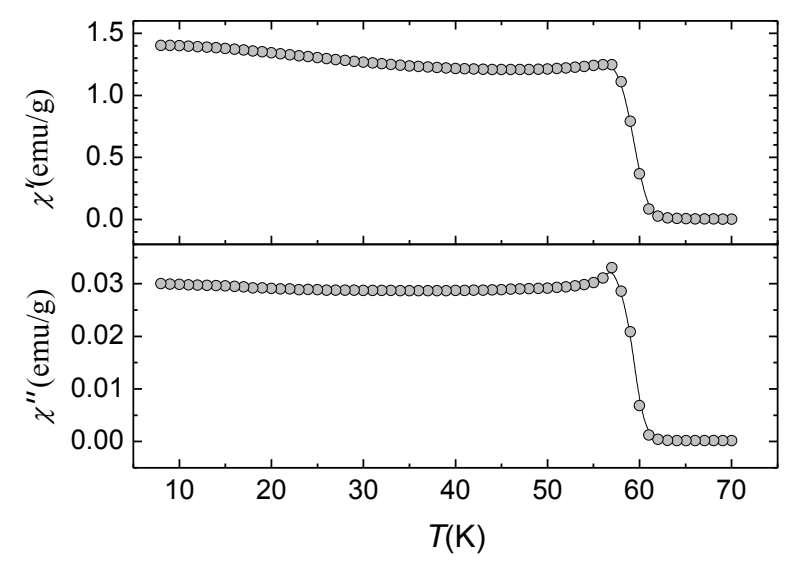

Fig. 2 AC Susceptibility $\chi(T)$ was measured in temperature range $\mathrm{T}=8-70 \mathrm{~K}$.

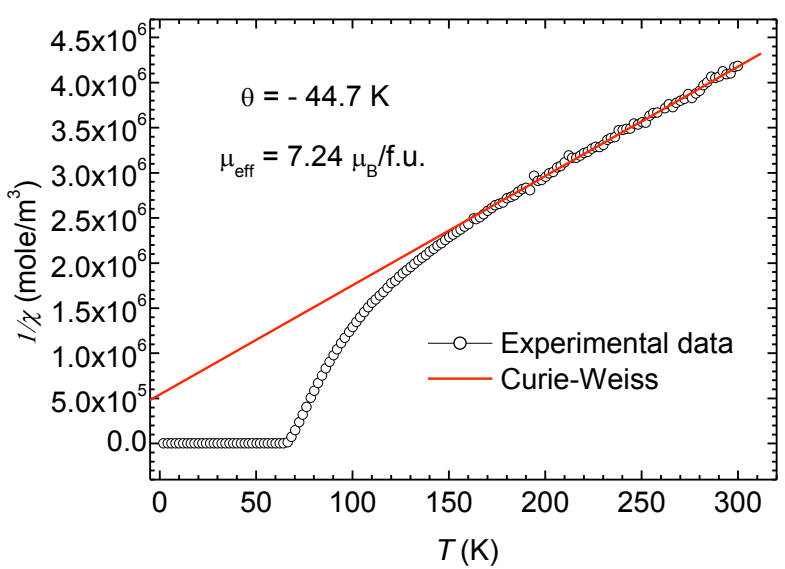

Fig.3. The inverse susceptibility together with the Curie Weiss law in $\mathrm{KMn}[\mathrm{Cr}(\mathrm{CN}) 6]$.

the superconducting transition of the high purity lead ( $T_{\mathrm{C}}$ $=7.19 \mathrm{~K}$ at ambient pressure). The pressure in the cell increases with increasing temperature in a nonlinear way, but this increase is comparable with the accuracy of the pressure determination, i.e. $\Delta p=0.03 \mathrm{GPa}$ in the measured temperature range, and can be neglected. Powder samples with a mass of about $5 \mathrm{mg}$ each were placed into a cylindrical holder $5 \mathrm{~mm}$ long and with a diameter of $2 \mathrm{~mm}$. The pressure was always applied at room temperature. The experiment started with the highest pressure, which was then gradually decreased in steps. There was no difference between the saturated magnetization $\mu_{\mathrm{s}}$ and the Curie temperature $T_{\mathrm{C}}$ determined at the ambient pressure before and after the pressure experiment.

\section{Results and discussion}

The X-ray powder diffraction pattern of investigated sample can be seen on Fig. $1 . \mathrm{KMn}\left[\mathrm{Cr}(\mathrm{CN})_{6}\right]$ crystallizes in cubic face centred system with lattice parameter $a=$ $10.786793 \AA$.

AC Susceptibility $\chi(\mathrm{T})$ was measured in modulation with the amplitude of $1 \mathrm{Oe}$ and frequency $\mathrm{f}=$ $2.5 \mathrm{~Hz}$. Measured data are shown in Fig. 2 and refer to a ferromagnetic material. High temperature data obeys the Curie-Weiss law yielding to negative value of paramagnetic Curie temperature $\theta=-44.7 \mathrm{~K}$, which point out to dominant antiferromagnetic superexchange interaction, and the effective magnetic moment $\mu_{\mathrm{eff}}=7.24$ $\mu_{\mathrm{B}}$ (see Fig. 3).

Magnetization saturates in very low magnetic fields at low temperatures, and above $\mu_{0} H=200 \mathrm{mT}$ is almost independent on the rising magnetic field. The remnant magnetization $\mu_{\mathrm{r}}$ and coercive field $H_{\mathrm{c}}$ for this material are close to zero [4]. As can be seen from Fig. 4, the magnetic isotherms change their shape with the applied pressure; magnetization saturates at higher magnetic field under pressure, but saturated magnetization $\mu_{\mathrm{s}}, \mu_{\mathrm{r}}$, and $H_{\mathrm{c}}$ are not significantly affected by pressure at low temperatures.

The applied pressure increases the strength of 


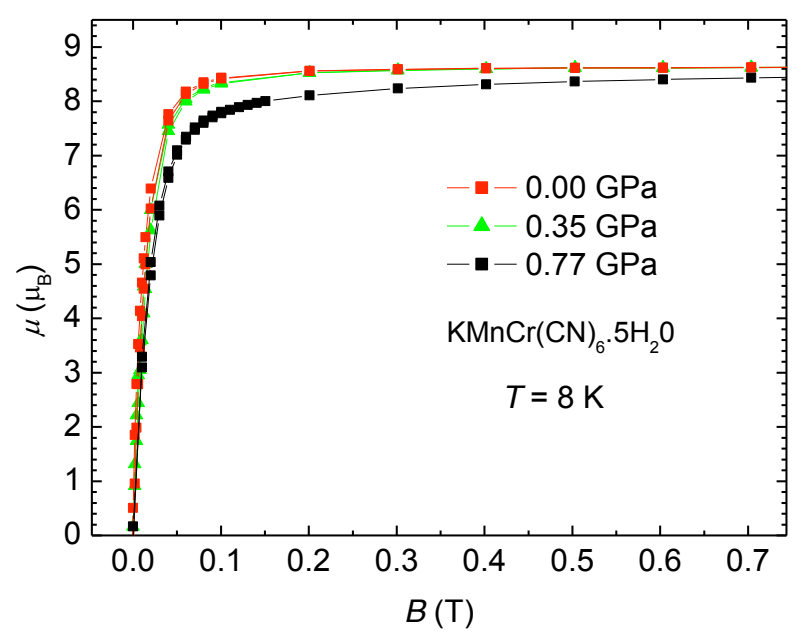

Fig.4. Magnetization $\mu\left(\mu_{0} H\right)$ measured at temperature $T$ $=8 \mathrm{~K}$ under different pressures. Below $T=8 \mathrm{~K}$ low field magnetization data are affected by the superconducting state of the $\mathrm{Pb}$ pressure sensor.

magnetic interaction in the system, as indicated by the increase of $T_{\mathrm{C}}$ (see Fig. 5). The transition to a magnetically ordered state is accompanied by a steep increase in $\mu(T)$. The Curie temperature $T_{\mathrm{C}}$ is defined as the inflection point of the $\mu(T)$ curve in this region i.e. as a minimum on $\mathrm{d} \mu(T) / \mathrm{d} T$. The applied pressure shifts $T_{\mathrm{C}}$ to higher temperatures almost linearly in this range of applied pressures. Applied pressure generates a step on $\mu(T)$ dependence in the vicinity of magnetic phase transition (fig.5.) and on $\mathrm{d} \mu(T) / \mathrm{d} T$ curve two minima occurs which we associate with two magnetic transitions . The pressure coefficients of the two magnetic phases are $\Delta T_{\mathrm{C} 1} / \Delta p=18.18 \mathrm{KGPa}^{-1}$ and $\Delta T_{\mathrm{C} 2} / \Delta p=26.62 \mathrm{KGPa}^{-1}$.

In conclusion, we summarize that the following pressure effect on magnetic properties of $\mathrm{KMn}\left[\mathrm{Cr}(\mathrm{CN})_{6}\right]$ compound was observed on both magnetization and thermomagnetic curves. Magnetization saturates at higher magnetic field under pressure, but saturated magnetization $\mu_{\mathrm{s}}$, remnant magnetization $\mu_{\mathrm{r}}$, and coercive force $\mathrm{H}_{\mathrm{C}}$ are not significantly affected by pressure at low temperatures. The Curie temperature $T_{\mathrm{C}}$ increases with the applied pressure, with coefficients $\Delta \mathrm{T}_{\mathrm{Cl}} / \Delta \mathrm{p}=18.18$ $\mathrm{KGPa}^{-1}$ and $\Delta \mathrm{T}_{\mathrm{C} 2} / \Delta \mathrm{p}=26.62 \mathrm{KGPa}^{-1}$. The additional magnetic phase was visible under pressure, the process was fully reversible.

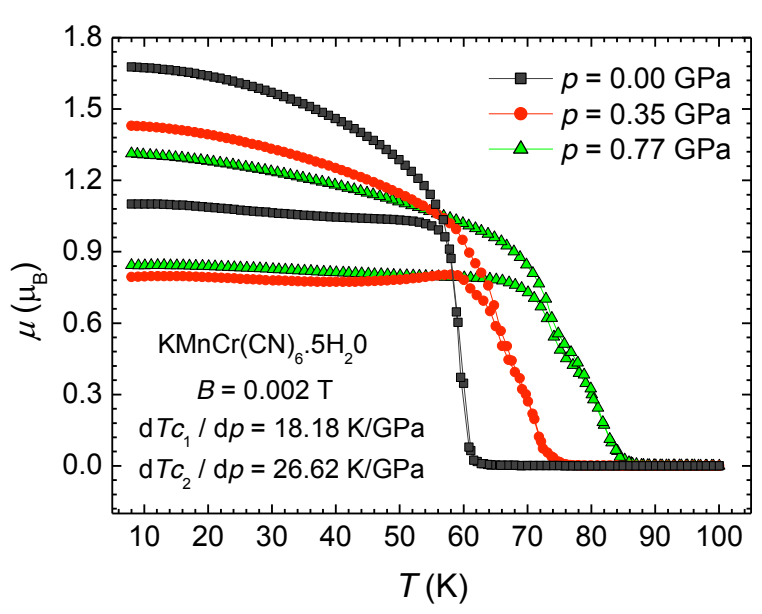

Fig.5. Magnetization $\mathrm{M}(\mathrm{T})$ measured under different pressures in $\mathrm{ZFC}$ and $\mathrm{FC}$ regimes in the magnetic field $\mu_{0} \mathrm{H}=2 \mathrm{mT}$.

\section{Aknowledgement}

This work was supported by the pro-jects VEGA 2/0057/10, ERDF EU under the contracts ITMS26220120005 and ESF EU ITMS626110230034.

\section{References}

1. L. Lawton, PhD thesis, School of Chemistry, University of Glasgow (2011)

2. M. Verdaguer and G. Girolami, Magnetism : Molecules to Materials V. ,WILEY-VCH Verlag, (2004), ISBN : 3-527-30665-XB., p.283-341

3. M. Zentková, M.Mihalik, J.Kováč, A. Zentko, Z.Mitróová ,V. Kavečanský, L.F. Kiss, phys.stat. sol. (b) 243, 272 ( 2006)

4. Z. Mitróová, S. Mat’aš , M.Mihalik, M. Zentková , Z.Arnold, J.Kamarád , Acta Physica Polonica A 113, 469 (2008)

5. D.Papanikolaou, W.Kosaka, S. Margadonna, H.Kagi, S.Ohkoshi, K. Prassides K J.Phys.Chem.C. 111, 8086 (2007)

6. K.Awaga, Taketoshi Sekine, Masaki Okawa, Wataru Fujita,Stephen M. Holmes, Gregory S. Girolami, Chemical Physics Letters 293, 352 (1998)

7. M.Zentkova,Z.Arnold,J.Kamarad,V.Kavecansky M..Lukacova,S.Matas, M Mihalik,Z.Mitroova, A. Zentko, J. Phys.: Condens. Matter 19, 266217 (2007)

8. D.S.Middlemiss, L.M. Lawton, Ch. C. Wilson, J.Phys.Condens. Matter 20, 335231( 2008) 